\title{
Decrease in Calcium Concentration Triggers Neuronal Retinoic Acid Synthesis during Homeostatic Synaptic Plasticity
}

\author{
Hui-Li Wang, Zhenjie Zhang, Maik Hintze, and Lu Chen \\ Stanford Institute of Neuro-Innovation and Translational Neuroscience and Department of Psychiatry and Behavioral Sciences, Stanford University School \\ of Medicine, Stanford, California 94305-5453
}

Blockade of synaptic activity induces homeostatic plasticity, in part by stimulating synthesis of all-trans retinoic acid (RA), which in turn increases AMPA receptor synthesis. However, the synaptic signal that triggers RA synthesis remained unknown. Using multiple activityblockade protocols that induce homeostatic synaptic plasticity, here we show that RA synthesis is activated whenever postsynaptic Ca ${ }^{2+}$ entry is significantly decreased and that RA is required for upregulation of synaptic strength under these homeostatic plasticity conditions, suggesting that $\mathrm{Ca}^{2+}$ plays an inhibitory role in $\mathrm{RA}$ synthesis. Consistent with this notion, we demonstrate that both transient $\mathrm{Ca}^{2+}$ depletion by membrane-permeable $\mathrm{Ca}^{2+}$ chelators and chronic blockage of L-type $\mathrm{Ca}^{2+}$-channels induces $\mathrm{RA}$ synthesis. Moreover, the source of dendritic $\mathrm{Ca}^{2+}$ entry that regulates $\mathrm{RA}$ synthesis is not specific because mild depolarization with $\mathrm{KCl}$ is sufficient to reverse synaptic scaling induced by L-type $\mathrm{Ca}^{2+}$-channel blocker. By expression of a dihydropyridine-insensitive $\mathrm{L}^{-t y p e} \mathrm{Ca}{ }^{2+}$ channel, we further show that RA acts cell autonomously to modulate synaptic transmission. Our findings suggest that, in synaptically active neurons, modest "basal" levels of postsynaptic $\mathrm{Ca}^{2+}$ physiologically suppress RA synthesis, whereas in synaptically inactive neurons, decreases in the resting $\mathrm{Ca}^{2+}$ levels induce homeostatic plasticity by stimulating synthesis of RA that then acts in a cell-autonomous manner to increase AMPA receptor function.

\section{Introduction}

Normal brain function requires that neurons operate within a dynamic range of overall activity, which is essential for optimal information coding. The processes that maintain network activity within this dynamic range are collectively called homeostatic plasticity (Turrigiano and Nelson, 2004; Davis, 2006). Synaptic scaling is a form of homeostatic plasticity in which synaptic strength is regulated in a multiplicative manner (i.e., stronger synapses are changed proportionally more than weaker synapses), primarily through changes in the abundance of postsynaptic AMPA-type glutamate receptors (Turrigiano et al., 1998; Thiagarajan et al., 2005). Previously we reported a critical role for all-trans retinoic acid (RA) in the induction of synaptic scaling (Aoto et al., 2008). Inhibition of action potential (AP) firing by tetrodotoxin (TTX), along with blockade of NMDA-type glutamate receptors by D-2-amino-5-phosphonovalerate (APV), stimulated synthesis of RA in neurons, and RA was both necessary and sufficient

Received Aug. 2, 2011; revised Sept. 29, 2011; accepted 0ct. 12, 2011.

Author contributions: H.-L.W. and L.C. designed research; H.-L.W., Z.Z., and M.H. performed research; H.-L.W. analyzed data; L.C. wrote the paper.

The research was supported by the David and Lucile Packard Foundation, the W. M. Keck Foundation, and National Institute of Mental Health Grants 1P50MH86403 and 1R01MH091193 (L.C.).

Correspondence should be addressed to Lu Chen, 265 Campus Drive, Room G1034B, Lorry Lokey Stem Cell Research Building, Stanford University, Stanford, CA 94305-5453. E-mail: luchen1@stanford.edu.

H.-L. Wang's present address: School of Biotechnology and Food Engineering, Hefei University of Technology, 193 Tunxi Road, Hefei, Anhui 230009, China.

DOI:10.1523/JNEUROSCI.3964-11.2011

Copyright $\odot 2011$ the authors $\quad 0270-6474 / 11 / 3117764-08 \$ 15.00 / 0$ to induce local translation of AMPA receptors in neuronal dendrites and to trigger synaptic scaling (Aoto et al., 2008). These findings placed RA into a key role in regulating synaptic strength. However, synaptic upscaling can be induced by several different manipulations. For example, a form of synaptic scaling induced by prolonged blockade of AP firing with TTX involves cell-wide, transcription-dependent changes (Turrigiano and Nelson, 2004). More recently, accumulating evidence supports the existence of a fast adaptive form of synaptic scaling that involves activation of protein synthesis locally in neuronal dendrites, allowing adjustment of synaptic strength at spatially discrete locations within a neuron (Ju et al., 2004; Sutton et al., 2006; Aoto et al., 2008). The latter form of synaptic scaling is induced by blockade of synaptic transmission mediated by a specific glutamate receptor type alone or concurrently with AP blockade (Turrigiano et al., 1998; Ju et al., 2004; Thiagarajan et al., 2005; Sutton et al., 2006). Although RA has been shown to regulate dendritic protein synthesis in an activity-dependent manner, it is unknown how general the role of RA is in homeostatic plasticity and what signal triggers RA synthesis.

In this study, we report that multiple manipulations that block glutamatergic synaptic transmission, alone or concurrently with AP blockade, stimulate RA synthesis. Synaptic scaling induced by such manipulations is mediated by synaptic insertion of GluA2lacking AMPA receptors and can be blocked by RA synthesis inhibitors. Moreover, simply blocking L-type calcium channels or chelating intracellular calcium strongly activates RA synthesis. Together, our findings suggest that RA is a general component in 
multiple forms of synaptic scaling, whose expression is mediated by local synthesis and synaptic insertion of GluA2-lacking AMPA receptors, and that removing repression of RA synthesis by calcium-dependent signaling mechanisms underlies activityregulated RA synthesis and synaptic upscaling.

\section{Materials and Methods}

Drugs and chemicals. The following drugs and chemicals were purchased from Sigma-Aldrich: RA, philanthotoxin-433 (PhTx), 4-diethylaminobenzaldehyde (DEAB), nifedipine, BAPTA-AM, and picrotoxin. TTX, APV, and 6-cyano-7-nitroquinoxaline-2,3-dione (CNQX) were purchased from Tocris Biosciences. EGTA-AM was purchased from Invitrogen.

Cell cultures and drug treatment. Primary hippocampal cultures were prepared from the brains of rats at embryonic day 22 and maintained in serum-free Neurobasal medium supplemented with B-27 and Glutamax (Invitrogen) for 2-3 weeks in vitro (Nam and Chen, 2005). Manipulations used to induce synaptic scaling in dissociated cultures include the following: TTX + APV $(1 \mu \mathrm{M}$ TTX + $100 \mu \mathrm{M} \mathrm{APV}, 24 \mathrm{~h}) ; \mathrm{CNQX}(10 \mu \mathrm{M}$, $24 \mathrm{~h}) ; \mathrm{TTX}+\mathrm{CNQX}(1 \mu \mathrm{M}$ TTX $+10 \mu \mathrm{M}$ CNQX, $24 \mathrm{~h})$, TTX $(1 \mu \mathrm{M}$, $48 \mathrm{~h}$ ), and nifedipine (10 $\mu \mathrm{M}, 24 \mathrm{~h})$. Stock solution of DEAB in DMSO was freshly made right before treatment, and $10 \mu \mathrm{M}$ DEAB was applied when indicated. Fifty-minute treatment of either $10 \mu \mathrm{M}$ BAPTA-AM or $10 \mu \mathrm{M}$ EGTA-AM was used to decrease the intracellular calcium level.

Retinoic acid response element assay. The $3 x$ RARE-EGFP reporter construct is as described previously (Aoto et al., 2008). Three copies of the retinoic acid response element (RARE) were placed upstream of a TK promoter driving EGFP. Dissociated cultures used for RARE imaging were transfected using Lipofectamine 2000 (Invitrogen) with a protocol described previously (Aoto et al., 2008), fixed with $4 \%$ paraformaldehyde (15 min, room temperature), and washed with PBS before mounting. Images were acquired and quantified as described previously (Nam and Chen, 2005) using an Olympus FV1000 BX61WI laser-scanning confocal microscope.

Electrophysiology. Whole-cell patch-clamp recordings were made at room temperature from 13 to $14 \mathrm{~d}$ in vitro (DIV) cultured neurons, with 4-6 $\mathrm{M} \Omega$ patch pipettes filled with an internal solution containing the following (in mM): $120 \mathrm{CsCl}, 2 \mathrm{MgCl}_{2}, 5 \mathrm{EGTA}, 10 \mathrm{HEPES}, 0.3 \mathrm{Na}_{3}$-GTP, and $4 \mathrm{Na}_{2}$-ATP, pH 7.35. Cultures were continuously superfused with external solution containing the following (in $\mathrm{mm}$ ): $100 \mathrm{NaCl}, 26$ $\mathrm{NaHCO}_{3}, 2.5 \mathrm{KCl}, 11$ glucose, $2.5 \mathrm{CaCl}_{2}, 1.3 \mathrm{MgSO}_{4}$, and $1.0 \mathrm{NaH}_{2} \mathrm{PO}_{4}$. For miniature EPSC (mEPSC) recording, TTX $(1 \mu \mathrm{M})$ and picrotoxin $(100 \mu \mathrm{M})$ were included in the external saline. Cells were held at $-60 \mathrm{mV}$. For PhTx recordings, $5 \mu \mathrm{M}$ PhTx or vehicle control was bath perfused for $10 \mathrm{~min}$ before recording. Miniature responses were analyzed with Mini Analysis Program from Synaptosoft.

Statistical analysis. Single-factor ANOVA was used for statistical analysis. Values are presented as mean \pm SEM in the figures.

\section{Results}

\section{RA synthesis is induced by multiple activity blockade} protocols producing homeostatic plasticity

We first examined whether RA synthesis is stimulated by activity blockade with different protocols that induce synaptic scaling (Turrigiano et al., 1998; Ju et al., 2004; Thiagarajan et al., 2005; Sutton et al., 2006). We chose four manipulations: blocking APs alone with TTX for 24 and $48 \mathrm{~h}$, blocking AMPA receptors with CNQX for $24 \mathrm{~h}$, or blocking both AP and AMPA receptors with TTX and CNQX, respectively, for $24 \mathrm{~h}$. Additionally, we included a 24-h TTX + APV treatment (blocking APs and NMDA receptors) as a positive control because of its well-characterized effects on RA synthesis (Aoto et al., 2008). All experiments were performed with cultured hippocampal neurons, which were transfected at 14 DIV with a plasmid containing multiple copies of a RARE driving transcription of GFP to measure RA levels by monitoring the GFP intensity (Aoto et al., 2008).

Consistent with our previous report, $24 \mathrm{~h}$ TTX + APV treatment significantly increased RA levels in neurons (Fig. 1A,B).
A
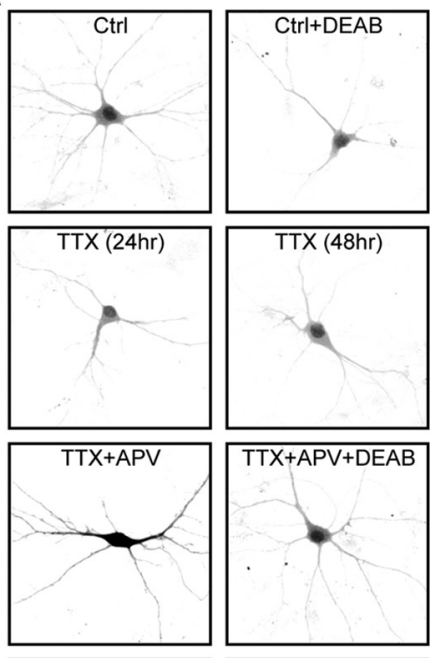

TTX (48hr)
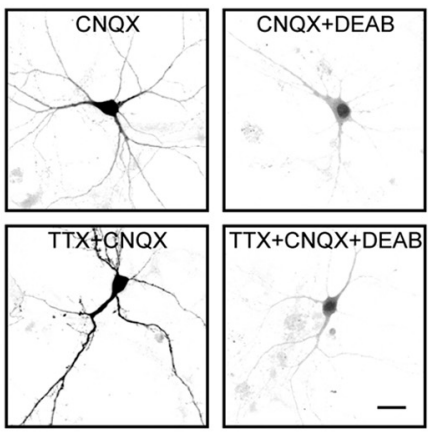

B

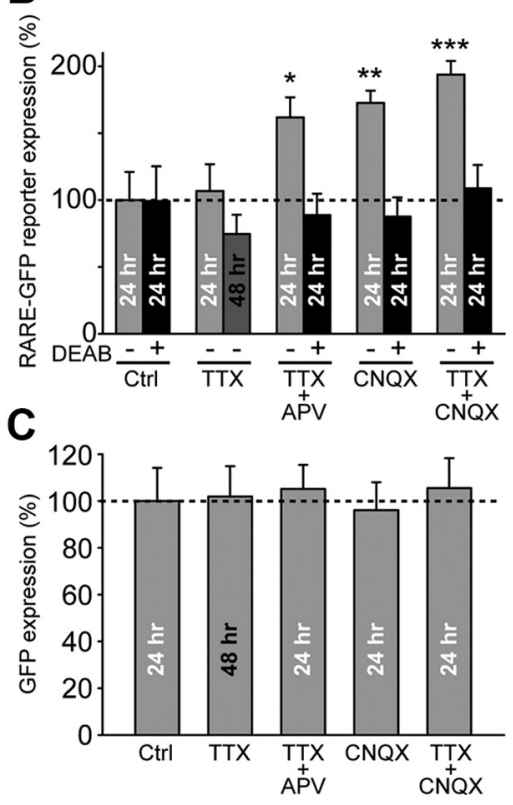

Figure 1. Prolonged blockade of synaptic transmission induces RA synthesis. $A$, Representative images of RARE-GFP reporter expression in neurons treated with different activity blockade protocols in the presence or absence of DEAB. Scale bar, $10 \mu \mathrm{m}$. $\boldsymbol{B}$, Quantification of RARE-GFP expression. Duration of treatments are as marked. DEAB was added together with activity-blocking drugs $(n=$ 12-30 from three independent experiments; ${ }^{*} p<0.05 ;{ }^{* *} p<0.005$; ${ }^{* * *} p<0.001$ ). C, Expression levels of a control GFP reporter lacking the RARE sequence under various activity blockade protocols. All treatments are $24 \mathrm{~h}$ except for TTX (48h) $(n=10-16)$. Ctrl, Control.

Blocking AMPA receptor-mediated synaptic transmission alone (CNQX, $24 \mathrm{~h}$ ) or together with AP blockade (TTX + CNQX, $24 \mathrm{~h}$ ) also significantly increased RA levels in neurons (Fig. $1 A, B)$. Interestingly, TTX alone applied for either 24 or $48 \mathrm{~h}$ did 
not induce RA synthesis (Aoto et al., 2008; Fig. $1 A, B)$, suggesting that blocking APs alone is insufficient for activating RA synthesis. All increases in RARE-GFP reporter expression were prevented by concurrent application of DEAB, an inhibitor of RA synthesis (Russo et al., 1988; Fig. 1A,B), confirming that the observed enhancement of GFP expression reflects an increase in RA synthesis. Additionally, expression of GFP from the same reporter plasmid lacking the RARE sequence failed to respond to activity blockade (Fig. 1C), suggesting that the increase in RARE-GFP expression is attributable to specific transcriptional activation by RA and not to a generally altered transcription or translation by activity blockade.

\section{$\mathrm{RA}$ is required for multiple forms of synaptic scaling}

We next recorded mEPSCs from neurons treated by activity blockade to evaluate changes in synaptic transmission. As expected, all four activity-blockade protocols tested ( $24 \mathrm{~h}$ treatment of TTX + APV, CNQX, TTX + CNQX, or 48 h of TTX) significantly increased mEPSC amplitudes (Fig. $2 A, B$ ). The mEPSC frequency was not significantly changed in any of the groups (Fig. 2B, 14 DIV groups). Addition of $\mathrm{DEAB}$ to the activity blockade mixture completely blocked the increases in mEPSC amplitudes induced by the TTX + APV, CNQX, or TTX + CNQX treatments (Fig. $2 B)$. However, the mEPSC amplitude increases induced by $48 \mathrm{~h}$ treatments with TTX alone were unaffected (Fig. 2B). This result, together with the RARE-GFP reporter expression result, demonstrates that RA is critically involved in synaptic scaling induced by blocking postsynaptic depolarization through glutamate receptor activation but that a second type of synaptic scaling induced by TTX alone uses an RAindependent mechanism.

RA is known as a transcriptional activator that functions during development. Recent evidence indicates that, in mature neurons, RA regulates protein synthesis in a transcription-independent manner and participates in synaptic scaling by activating local synthesis of the AMPA receptor GluA1 subunit, resulting in postsynaptic insertion of $\mathrm{Ca}^{2+}$ permeable homomeric GluA1-containing AMPA receptors (Aoto et al., 2008; Maghsoodi et al., 2008; Poon and Chen, 2008). We therefore examined the subunit composition of the newly inserted synaptic AMPA receptors induced by different synaptic scaling protocols. The increase in mEPSC amplitude in neurons treated with TTX + APV, CNQX, or TTX + CNQX was fully reversed by PhTx, a blocker of GluA2-lacking AMPA receptors (Fig. 2C), indicating that the increase in synaptic strength is caused by synaptic insertion of AMPA receptors lacking GluA2. Synaptic scaling induced by a $48 \mathrm{~h}$ treatment with TTX alone, however, was insensitive to PhTx 0.001). Ctrl, Control.
A
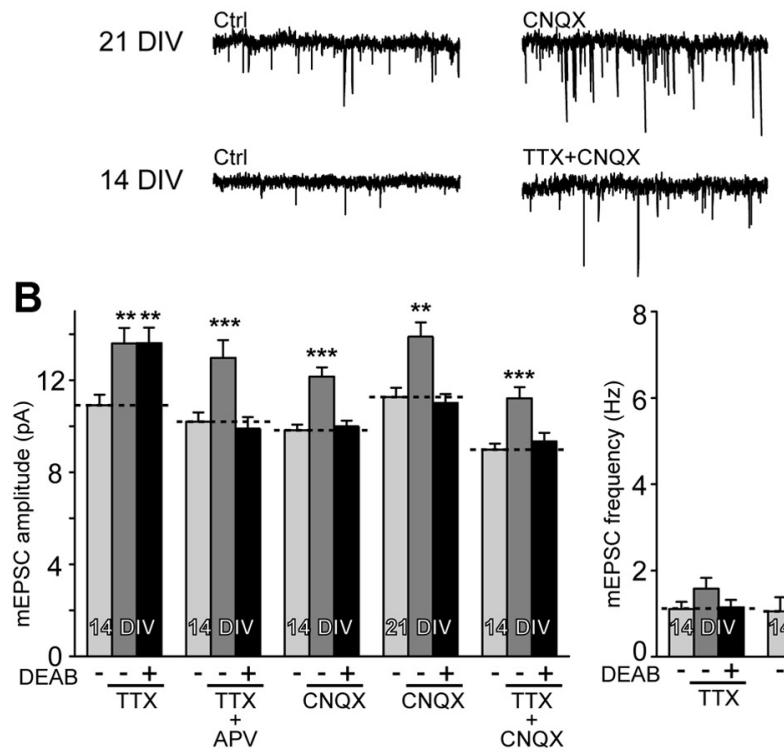
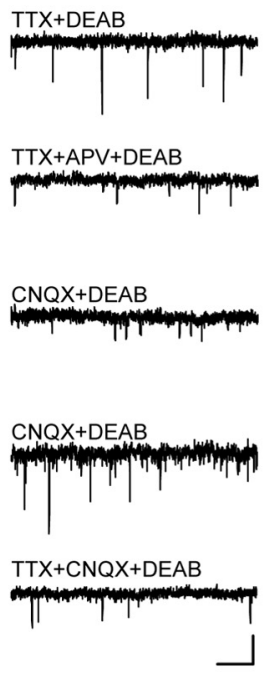

$\mathrm{CNQX}+\mathrm{DEAB}$
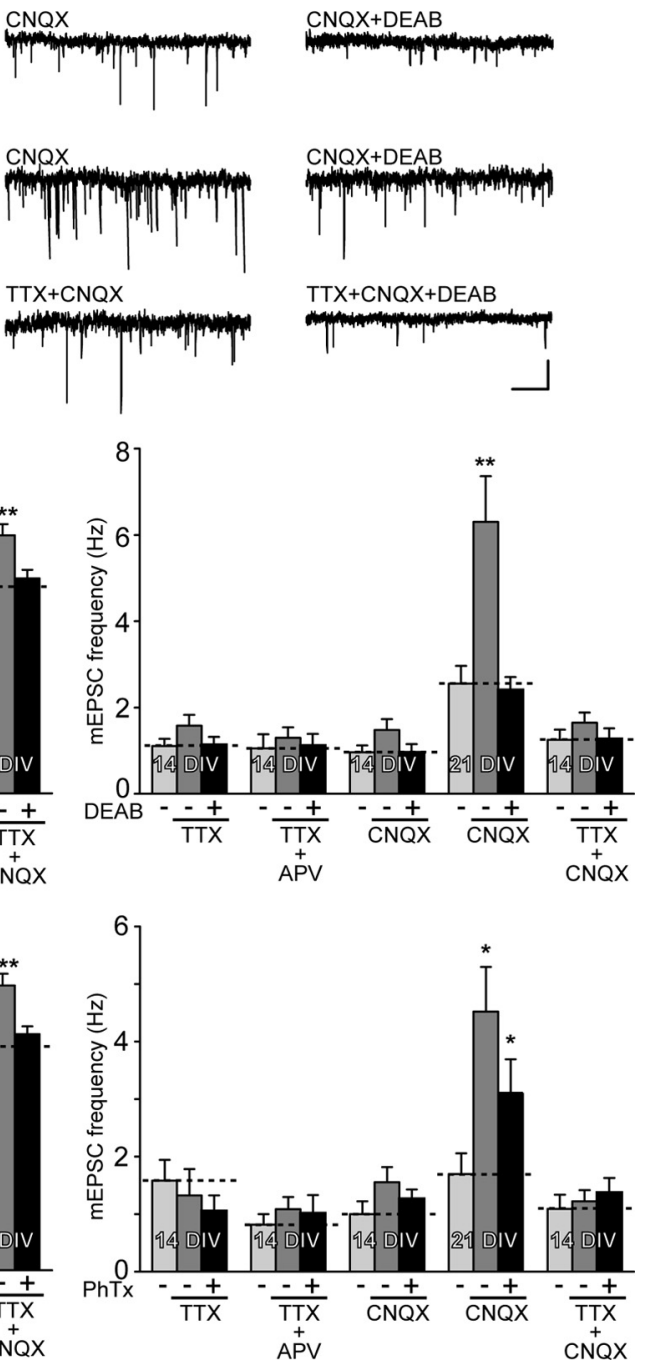

Figure 2. RA dependence of synaptic scaling induced by different activity blockade protocols. $A$, Representative traces of $m E P S C s$ from neurons treated with different activity blockade protocols in the presence or absence of DEAB. All treatments are $24 \mathrm{~h}$ except for TTX treatment, which was $48 \mathrm{~h}$. The age of the cultures are as indicated. Calibration: $10 \mathrm{pA}, 1 \mathrm{~s}$. B, Quantification of $\mathrm{mEPSC}$ amplitude and frequency from neurons treated with different activity blockade protocols in the presence or absence of $\mathrm{DEAB}\left(n=23-39\right.$ from $>3$ independent experiments; $\left.{ }^{* *} p<0.005 ;{ }^{* * *} p<0.001\right)$. C, Sensitivity of scaled mEPSC amplitude and frequency to acute application of $\operatorname{PhTx}(5 \mu \mathrm{M})\left(n=20-38\right.$ from $>3$ independent experiments; ${ }^{*} p<0.05 ;{ }^{* *} p<0.005 ;{ }^{* * *} p<$

treatment, consistent with previous reports showing that GluA1/ GluA2 heteromeric AMPA receptors are incorporated into the synapses in response to this treatment (Wierenga et al., 2005).

Previous studies showed that synaptic scaling induced by prolonged blockade of AMPA receptors produces an increase in both mEPSC amplitude and frequency, with the latter effect mediated by postsynaptic synthesis and release of brain-derived neurotrophic factor (BDNF) (Thiagarajan et al., 2005; Jakawich et al., 2010; Lindskog et al., 2010). In our experiments, we used cultured hippocampal neurons that are younger (14 DIV) than those in the published studies and observed only a trend of an increase in 
mEPSC frequency that was not statistically significant (Fig. $2 B, C)$. Given that BDNF expression in the brain increases steeply between postnatal days 15 and 20 (Schoups et al., 1995), we examined synaptic scaling induced by a $24 \mathrm{~h}$ CNQX treatment in older cultured hippocampal neurons at 21 DIV. Indeed, blocking AMPA receptors for $24 \mathrm{~h}$ in these older neurons induced a significant increase in both mEPSC amplitude and frequency (Fig. $2 B, C)$. Consistent with a previous report (Jakawich et al., 2010), the increase in mEPSC amplitude was completely reversed by blockers specific for GluA2-lacking receptors such as PhTx, but the mEPSC frequency remained significantly higher than in control neurons even in the presence of $\mathrm{PhTx}$ (Fig. 2C). Importantly, this form of synaptic scaling, which involves changes in both presynaptic and postsynaptic compartments, is blocked by DEAB (Fig. $2 B$ ), indicating that RA synthesis surprisingly is also required for inducing these increases in mEPSC frequency, which is surprising because such increases are generally thought to represent a presynaptic effect.

\section{Suppression of $\mathrm{Ca}^{2+}$ signaling is sufficient to induce RA synthesis}

Why is RA synthesis selectively induced by glutamate receptor blockade but not by abrogating AP generation? Although GluA2containing AMPA receptors are virtually $\mathrm{Ca}^{2+}$ impermeable, activation of AMPA receptors is expected to induce sufficient depolarization to increase $\mathrm{Ca}^{2+}$ influx through $\mathrm{Ca}^{2+}$-permeable NMDA receptors and L-type $\mathrm{Ca}^{2+}$ channels. We therefore hypothesized that, under resting conditions, RA synthesis is suppressed by postsynaptic $\mathrm{Ca}^{2+}$ influx induced by spontaneous miniature excitatory synaptic events or by network activity.

To test this hypothesis, we applied the membrane-permeable $\mathrm{Ca}^{2+}$ chelators BAPTA-AM and EGTA-AM to cultured neurons expressing the RARE-GFP reporter. Short (50 min) treatments of neurons with either BAPTA-AM or EGTA-AM dramatically stimulated RARE-regulated GFP expression (Fig. 3) but not GFP expression from a control plasmid lacking RAREs (normalized to control treatment: BAPTA-AM, $99 \pm 11.4 \%, n=16$; EGTA-AM, $119 \pm 6.7 \%, n=12 ; p>0.2)$. The RARE-dependent increase in GFP expression induced by the $\mathrm{Ca}^{2+}$ chelators was blocked by DEAB (Fig. 3), confirming that decreasing the postsynaptic $\mathrm{Ca}^{2+}$ concentration induces RA synthesis.

To further test the regulation of RA synthesis by postsynaptic $\mathrm{Ca}^{2+}$ in the context of synaptic scaling, we examined whether RA synthesis can be activated by blockade of L-type $\mathrm{Ca}^{2+}$ channels. Primarily localized in the soma and dendrites (Hell et al., 1993), L-type $\mathrm{Ca}^{2+}$ channels significantly contribute to dendritic $\mathrm{Ca}^{2+}$ signaling. Prolonged blockade of L-type $\mathrm{Ca}^{2+}$ channels has been shown to mimic the effects of AMPA receptor blockade and induce synaptic scaling (Thiagarajan et al., 2005). Indeed, treating neurons for $24 \mathrm{~h}$ with nifedipine, an L-type $\mathrm{Ca}^{2+}$ channel blocker, enhanced RARE-GFP reporter expression (Fig. 3) but not expression of the control GFP reporter (normalized to control treatment: $96.3 \pm 12.3 \%, n=10, p>0.8)$. DEAB again blocked the increase in the RARE-GFP signal (Fig. 3), suggesting that the increase is specifically attributable to RA synthesis but not a global increase in transcription or translation triggered by reduced $\mathrm{Ca}^{2+}$ levels in the neuron (Pang et al., 2010). Consistent with previous reports (Thiagarajan et al., 2005), $24 \mathrm{~h}$ nifedipine treatment in our hands also significantly increased excitatory synaptic transmission (Fig. 4). Importantly, DEAB treatment blocked nifedipine-induced synaptic scaling (Fig. 4A), further supporting the hypothesis that RA mediates the nifedipineinduced increase in synaptic strength. Consistent with the role of
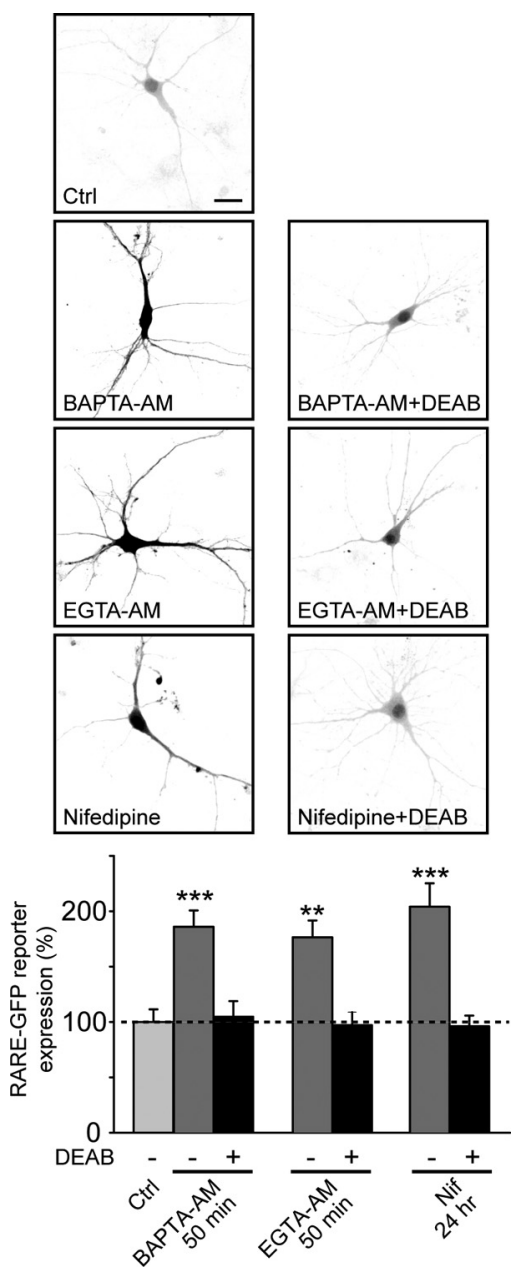

Figure 3. Calcium-dependent regulation of RA synthesis. Representative images (top) and quantification (bottom) of RARE-GFP reporter expression in neurons treated with BAPTA-AM (50 min), EGTA-AM (50 min), or nifedipine $(24 \mathrm{~h})$ in the presence or absence of DEAB ( $n=$ 21-30 from $>3$ independent experiments; ${ }^{* *} p<0.005 ;{ }^{* * *} p<0.001$ ). Scale bar, $10 \mu \mathrm{m}$. Ctrl, Control.

RA in activating dendritic GluA1 synthesis and promoting synaptic insertion of GluA1-containing homomeric AMPA receptors, PhTx reversed the increase in mEPSC amplitude seen in nifedipine-treated neurons (Fig. 4B). Somewhat surprisingly, blocking $\mathrm{Ca}^{2+}$ entry through NMDA receptors alone with APV failed to induce synaptic scaling even after $48 \mathrm{~h}$ (Fig. 5), suggesting that, under basal conditions when spontaneous AP firing can occur, $\mathrm{Ca}^{2+}$ enters postsynaptic compartment mostly through non-NMDA receptor-mediated sources. This observation is consistent with the role of NMDA receptors as coincidence detectors and with the finding that most $\mathrm{Ca}^{2+}$ entry in response to release of presynaptic glutamate alone (without pairing with postsynaptic AP) is mediated by voltage-gated $\mathrm{Ca}^{2+}$ channels, with NMDA receptor activation accounting for $<20 \%$ of the total $\mathrm{Ca}^{2+}$ entry (Schiller et al., 1998).

The fact that blocking $\mathrm{Ca}^{2+}$ entry through either synaptic glutamate receptors or L-type $\mathrm{Ca}^{2+}$ channels induces RA synthesis suggests that the source of $\mathrm{Ca}^{2+}$ entry is not important for suppression of RA synthesis and that de-repression of RA synthesis requires only a partial decrease in $\mathrm{Ca}^{2+}$ entry because blocking AMPA receptor or L-type $\mathrm{Ca}^{2+}$ channels is sufficient for stimulating RA synthesis. To further test this hypothesis, we asked whether we could block nifedipine-induced synaptic scaling by 

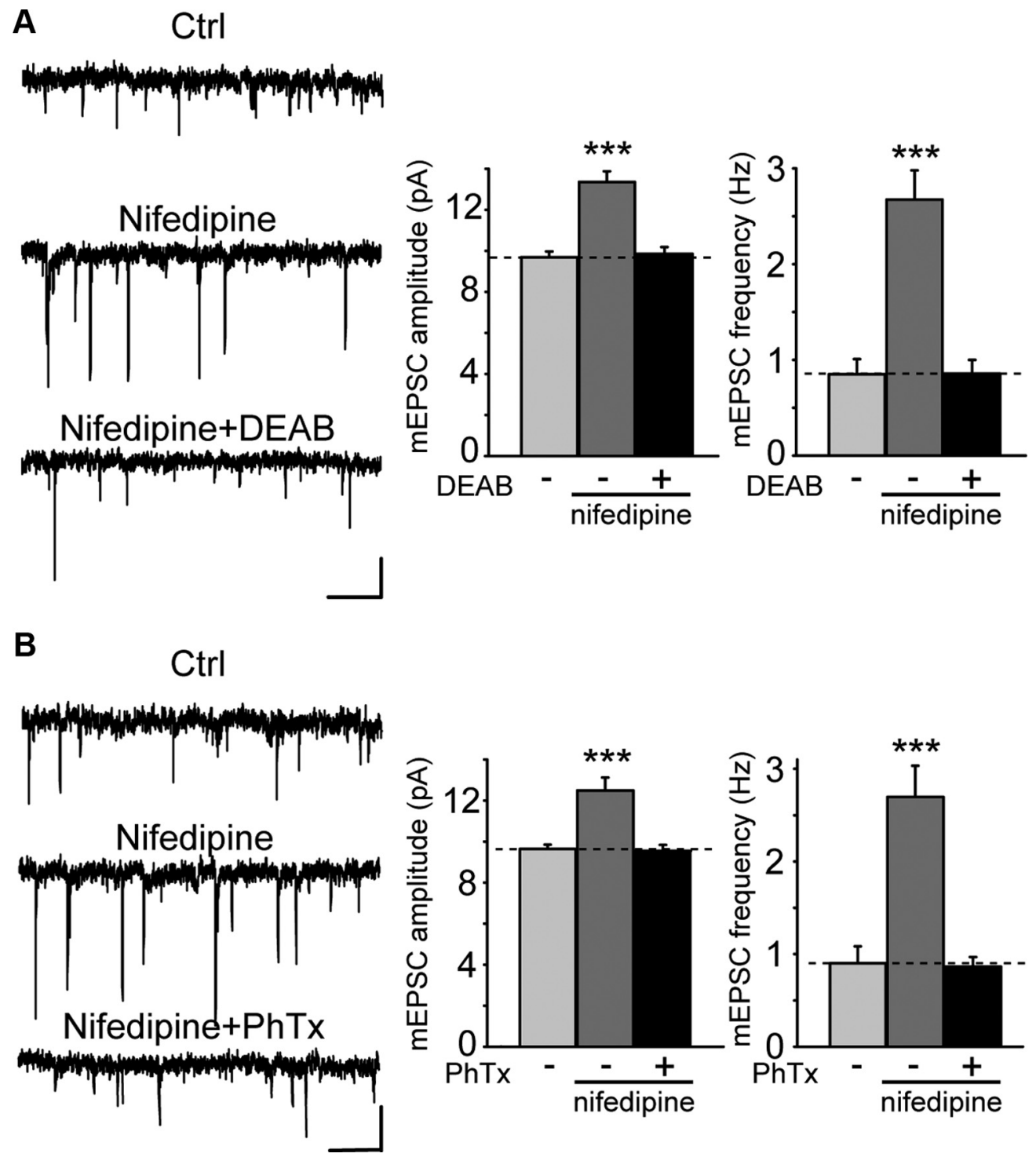

Figure 4. RA mediates nifedipine-induced synaptic scaling. $A$, Sensitivity of $24 \mathrm{~h}$ nifedipine-induced synaptic scaling to RA synthesis blocker DEAB ( $n=21-30$ from 3 independent experiments; ${ }^{*} p<0.05$ ). Calibration: $10 \mathrm{pA}, 1$ s. B, Sensitivity of $24 \mathrm{~h}$ nifedipine-induced synaptic scaling to PhTx ( $n=14-17$ from 3 independent experiments; $\left.{ }^{* * *} p<0.001\right)$. Calibration: $10 \mathrm{pA}, 1 \mathrm{~s}$. Ctrl, Control.

depolarizing neurons using a moderate increase in the external $\mathrm{K}^{+}$concentration, which would be expected to activate $\mathrm{Ca}^{2+}$ influx via other channels. Indeed, depolarizing neurons with 15 $\mathrm{mm}$ external $\mathrm{KCl}$ completely prevented synaptic scaling induced by nifedipine (Fig. 6), although $\mathrm{KCl}$ treatment alone did not alter the mEPSC amplitude or frequency (Fig. 6).

\section{RA-mediated synaptic scaling acts cell autonomously}

The question arises whether RA, which enhances AMPA receptor synthesis and insertion in neurons, is produced in the silenced neurons and acts cell autonomously in the same neuron. For example, it is possible that RA is synthesized in neurons with reduced synaptic transmission and secreted in a paracrine manner to neighboring neurons to effect changes in synaptic strength. It is also possible that RA is synthesized in astrocytes and diffuses into neurons to stimulate AMPA receptor function. This possibility is raised by the fact that astrocytes express AMPA and NMDA receptors (Lin and Bergles, 2004; Ge et al., 2006; Cahoy et al., 2008), L-type $\mathrm{Ca}^{2+}$ channels (Cahoy et al., 2008), and RA biosynthetic enzymes (Wang et al., 2011). Because all of our pharmacological manipulations affect neurons and glia cells alike, these manipulations could have induced RA synthesis in astrocytes instead of neurons.

To address this important question, we made use of a mutant, dihydropyridine (DHP)-insensitive L-type $\mathrm{Ca}^{2+}$-channel, $\alpha_{1 \mathrm{C} /}$ DHPi, that contains two amino acid substitutions (T1039Y and Q1043M) that render it insensitive to nifedipine blockade (Hockerman et al., 2000). We sparsely transfected cultured neurons with a plasmid encoding $\alpha_{1 \mathrm{C} / \mathrm{DHPi}}$ and coexpressing GFP (for identification of transfected neurons) and treated the neurons with nifedipine for $24 \mathrm{~h}$. Given our low transfection efficiency $(<5 \%)$, only a few neurons expressed the nifedipine-resistant $\mathrm{Ca}^{2+}$ channel, whereas most of surrounding neurons and glial cells were untransfected and therefore susceptible to nifedipine blockade. If RA synthesized in these untransfected neurons and glial cells diffused into $\alpha_{1 \mathrm{C} / \mathrm{DHPi}^{-}}$transfected neurons, synaptic scaling should still be induced in the $\alpha_{1 \mathrm{C} / \mathrm{DHPi}^{-}}$-expressing neurons by chronic nifedipine treatments. 

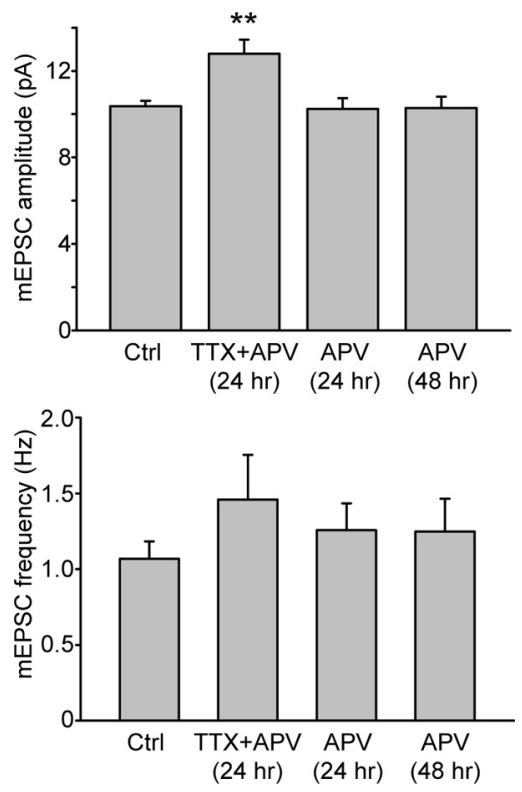

Figure 5. Blocking NMDA receptor alone does not induce synaptic scaling. Treatments as indicated are done in 14 DIV neurons. No increase in mEPSC amplitude or frequency was observed under 24 or $48 \mathrm{~h}$ of APV treatment. TTX + APV treatment was used as a positive control in the same batches of cultures $(n=11-29 ; * * p<0.005)$. Ctrl, Control.
This result strongly suggests that, during synaptic scaling, RA is most likely induced locally in silenced neurons and primarily acts cell autonomously to stimulate AMPA receptor synthesis and insertion.

\section{Discussion}

Our previous work demonstrated that RA directly potentiates excitatory synaptic transmission by increasing the postsynaptic AMPA receptor response in a synapse, that RA synthesis is induced during synaptic silencing to mediate homeostatic plasticity, and that blocking RA synthesis prevents the increase in AMPA receptor responses induced by synaptic silencing (Aoto et al., 2008; Soden and Chen, 2010). These results raised three major questions. What postsynaptic signaling pathway mediates the induction of RA synthesis during synaptic silencing? Is RA synthesized in the "silenced" neurons or in glia? If synthesized in neurons, does RA act cell autonomously in the neuron in which it is synthesized, or does it act diffusely in a paracrine manner?

To address these questions, we here examined which of the multiple forms of synaptic scaling previously described (Turrigiano et al., 1998; Ju et al., 2004; Thiagarajan et al., 2005; Sutton et al., 2006) involves stimulation of RA synthesis. Strikingly but consistent with previous data, we find that the various protocols that lead to synaptic scaling can be classed into two groups. The first group (RA-independent) involves chronic treatment of neurons with TTX alone, which results in an increase in AMPA response sizes as measured by the amplitude of mEPSCs without inducing RA synthesis. This increase is insensitive to either the RA synthesis inhibitor DEAB or the AMPA receptor blocker PhTx that only acts on GluA2lacking receptors (Figs. 1, 2). The second RA-dependent group involves chronic treatment of neurons with either a combination of TTX and APV, with CNQX alone, or with CNQX in combination with TTX, which induced RA synthesis and produced an increase in AMPA receptor response that was blocked by either DEAB or PhTx (Figs. 1, 2).

In considering what differentiates the two classes of synaptic scaling mechanisms, we noted that TTX treatment alone does not decrease the $\mathrm{Ca}^{2+}$ influx into postsynaptic neurons to below the level induced by miniature synaptic transmission, whereas all other treatments are expected to reduce $\mathrm{Ca}^{2+}$ influx below that level. Thus, we examined whether simply lowering the neuronal $\mathrm{Ca}^{2+}$ levels using membrane-permeable $\mathrm{Ca}^{2+}$ chelators would induce RA synthesis and observed a strong stimulation of RA synthesis that was blocked by DEAB (Fig. 3). Next, we asked whether blocking L-type $\mathrm{Ca}^{2+}$ channels with nifedipine could also achieve this effect and found that chronic nifedipine treatments indeed induced RA synthesis and increased the mEPSC amplitude (Figs. 3, 4).

Recordings of mEPSCs revealed that, whereas untransfected neurons showed significant increases in both the amplitude and frequency of mEPSCs, transfected neurons expressing $\alpha_{1 \mathrm{C} / \mathrm{DHPi}}$ exhibited no synaptic scaling (Fig. 7). Thus, RA synthesized in neighboring neurons and glial cells does not seem to be sufficient to support synaptic scaling via diffusion, at least in dissociated cultures.
The results described in Figures 1-4 suggest a simple and straightforward mechanism mediating RA-dependent synaptic scaling induced by synaptic silencing (Fig. 8). Under normal conditions (no activity blockade), there are two main processes leading to a rise in dendritic $\mathrm{Ca}^{2+}$ levels. The first process involves 
activation of synaptic AMPA receptors, which causes depolarization of the postsynaptic membrane. Subsequent opening of voltage-dependent $\mathrm{Ca}^{2+}$ channels (VDCCs) and to a lesser extent synaptic NMDA receptors induces large amounts of $\mathrm{Ca}^{2+}$ entry into dendrites (the AMPAR-NMDAR and the AMPAR-VDCC pathways). The second process is activation of dendritic VDCCs by direct postsynaptic neuronal spiking (the AP-VDCC pathway). When one and/or the other process operate, RA synthesis is completely shut off. When AP firing is blocked by TTX, the AP-VDCC pathway is blocked, but a significant amount of $\mathrm{Ca}^{2+}$ can still enter through the AMPARNMDAR and the AMPAR-VDCC pathways as a result of preserved miniature synaptic transmission, and RA synthesis remains suppressed. Additional reduction of dendritic $\mathrm{Ca}^{2+}$ levels by addition of APV or CNQX on top of TTX triggers RA synthesis (Fig. 8). Alternatively, nifedipine alone, which blocks most if not all $\mathrm{Ca}^{2+}$ influx through dendritic VDCCs, effectively triggers RA synthesis by inhibiting both AMPAR-VDCC and APVDCC pathways. Although synaptic NMDAR activation does contribute to dendritic $\mathrm{Ca}^{2+}$ levels, its contribution is limited unless both presynaptic and postsynaptic neurons fire together, a coincidence that is rare in normal neural networks in culture. This explains the lack of effects of APV-alone treatment on RA synthesis. In summary, the amount of postsynaptic $\mathrm{Ca}^{2+}$ influx mediated by miniature synaptic events suppresses RA synthesis, whereas under conditions of reduced $\mathrm{Ca}^{2+}$ influx, RA synthesis is stimulated. Because partial lowering of $\mathrm{Ca}^{2+}$ influx by blocking either glutamate receptors or L-type $\mathrm{Ca}^{2+}$ channels is sufficient to de-repress RA synthesis, RA synthesis appears to be tightly regulated by $\mathrm{Ca}^{2+}$. The overall mechanism proposed here (Fig. 8) was further confirmed by the finding that the increase in AMPA receptor responses induced by nifedipine could be prevented by chronic moderate depolarization of neurons using $15 \mathrm{mM} \mathrm{KCl}$, which enhances $\mathrm{Ca}^{2+}$ influx via alternative routes (e.g., NMDA receptors and other types of $\mathrm{Ca}^{2+}$ channels; Fig. 6). The parsimonious nature of the mechanism of regulating synaptic scaling via RA proposed suggests a novel $\mathrm{Ca}^{2+}$-dependent signaling pathway in neurons in which RA plays a central role (Fig. 8).

How does $\mathrm{Ca}^{2+}$ signaling regulate RA synthesis? RA has long been known to play a critical role in early brain development, but the embryonic brain is devoid of RA synthesis. Instead, during early development, the brain is flanked by two regions of extremely high expression of retinal dehydrogenase (RALDH, a major enzyme in RA synthesis) along the rostrocaudal axis (Smith et al., 2001; Niederreither et al., 2002), which provides the
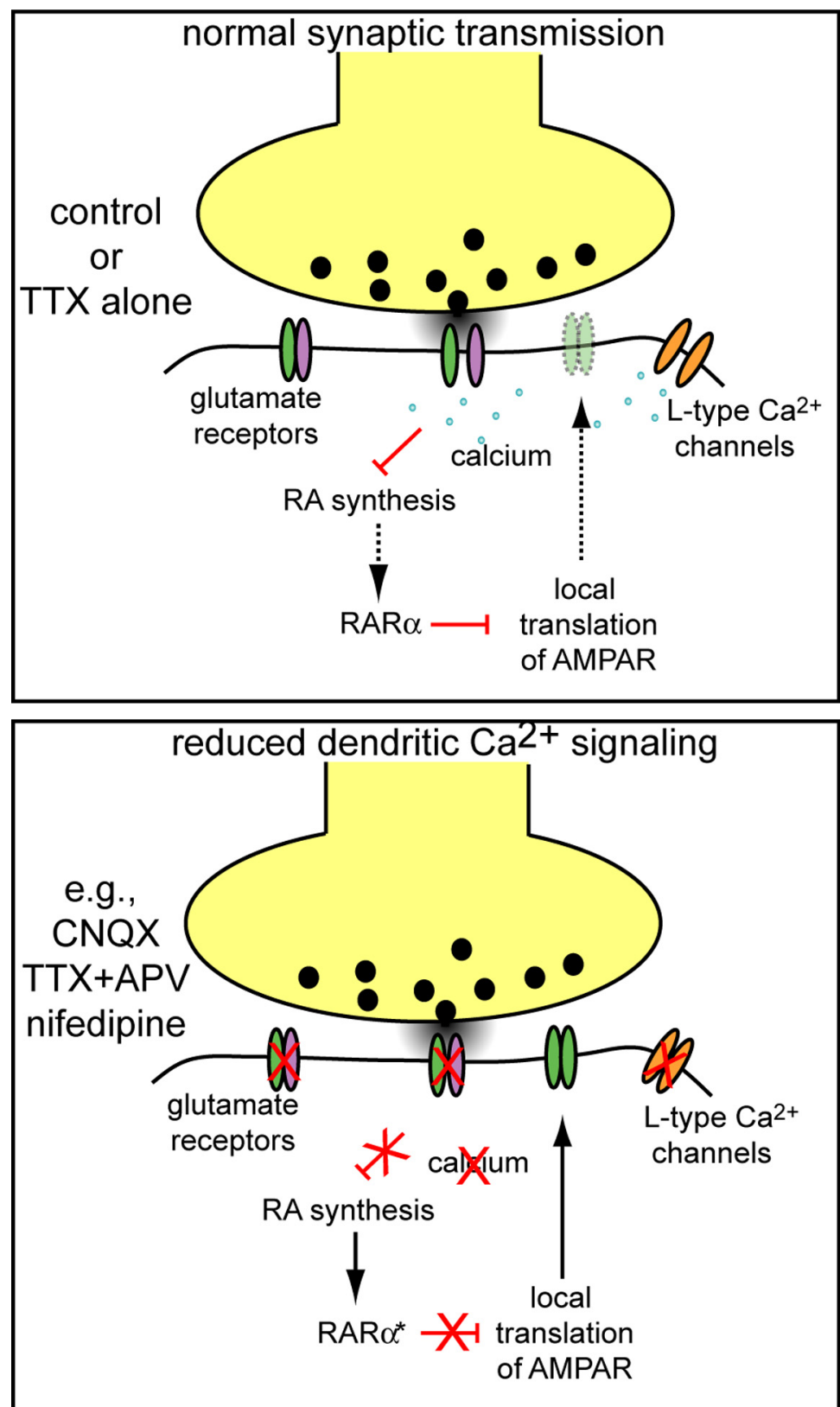

Figure 8. A model for activity-dependent RA synthesis in neurons. Normal synaptic transmission, via activation of glutamate -type $\mathrm{Ca}^{2+}$ channels lowers dendritic L-type $\mathrm{Ca}^{2+}$ influx and de-represses RA synthesis, which leads to translational activation and synaptic insertion of AMPARs in silenced neurons. RAR, RA receptor.

RA gradient required for the morphogenesis of the early hindbrain (Gavalas and Krumlauf, 2000). This situation is strikingly different from the adult brain in which RA is synthesized almost exclusively within the brain (Werner and Deluca, 2002), and the rate of RA synthesis is very high, twice the rate as in the retinoid-rich liver (Dev et al., 1993). It is generally believed that RALDHs are critical determinants for the sites of RA action (Wagner et al., 2002), making them attractive candidates subjected to modulation by activity. However, very little information is available regarding how neuronal activity may affect enzymatic activities of RALDHs. Additional investigation is required to uncover the components of this novel signaling pathway.

Interestingly, the increase in postsynaptic AMPA receptors produced by RA specifically stimulates the local synthesis and synaptic 
insertion of $\mathrm{Ca}^{2+}$-permeable GluA2-lacking AMPA receptors. Given the negative regulation of RA synthesis by dendritic $\mathrm{Ca}^{2+}$, it is conceivable that activation of $\mathrm{Ca}^{2+}$-permeable AMPA receptors serves as a negative feedback regulation for RA synthesis and therefore allows rapid stabilization of synaptic strength after homeostatic adjustment. The connection between decreased postsynaptic $\mathrm{Ca}^{2+}$ and RA synthesis suggests that RA signaling has wide implications for regulating synaptic strength and may have a broad impact on synaptic function, because synaptic $\mathrm{Ca}^{2+}$ signaling and dendritic protein synthesis are also important for use-dependent forms of synaptic plasticity (e.g., Hebbian-type plasticity).

The dependence of RA synthesis on nifedipine-sensitive L-type $\mathrm{Ca}^{2+}$ channels enabled us to test whether RA is produced locally in silenced neurons and whether the RA thus produced acts cell autonomously in the same neuron or provides a diffusible signal to surrounding neurons. When we expressed an L-type $\mathrm{Ca}^{2+}$ channel mutant that is nifedipine resistant in a small subset of neurons in our culture and analyzed nifedipine-induced synaptic scaling in these neurons, we found that synaptic scaling was blocked, despite the fact that all surrounding neurons scaled. This result indicates that RA is synthesized locally in a neuron and acts primarily in the same neuron to induce synaptic AMPA receptor synthesis. The result was surprising given the diffusible nature of RA and its ability to regulate synaptic strength when added to the culture medium at high concentrations (Aoto et al., 2008). Although the present data indicate that the RA levels produced physiologically in a neuron do not suffice for a long-range diffusible signal, because neuronal density is lower in dissociate culture systems than in vivo, our result does not exclude the possibility that, in densely packed tissues, RA may diffuse between neuronal cell bodies and dendrites.

In suggesting a general cell-autonomous role for RA in regulating synaptic strength in neurons, our data also raise a series of new questions. At present, little is known about the physiological importance of homeostatic plasticity, and we do not know under what conditions in vivo RA signaling modulates neural circuits, a question that will require sophisticated mouse genetics and pharmacology experiments in vivo to address. Another important question concerns the changes in mEPSC frequency that we observed during synaptic scaling consistent with previous papers (Thiagarajan et al., 2005; Jakawich et al., 2010). Because these changes may depend on BDNF as a secreted signal (Jakawich et al., 2010) but as we show here are also RA dependent, it may be possible that RA not only stimulates AMPA receptor synthesis and insertion but also BDNF secretion, which then takes the initially cell-autonomous action of RA to the presynaptic partners that send their inputs to the RA-synthesizing neuron. This exciting possibility broadens the possible scope of RA functions and again will involve sophisticated approaches to test.

\section{References}

Aoto J, Nam CI, Poon MM, Ting P, Chen L (2008) Synaptic signaling by alltrans retinoic acid in homeostatic synaptic plasticity. Neuron 60:308-320.

Cahoy JD, Emery B, Kaushal A, Foo LC, Zamanian JL, Christopherson KS, Xing Y, Lubischer JL, Krieg PA, Krupenko SA, Thompson WJ, Barres BA (2008) A transcriptome database for astrocytes, neurons, and oligodendrocytes: a new resource for understanding brain development and function. J Neurosci 28:264-278.

Davis GW (2006) Homeostatic control of neural activity: from phenomenology to molecular design. Annu Rev Neurosci 29:307-323.

Dev S, Adler AJ, Edwards RB (1993) Adult rabbit brain synthesizes retinoic acid. Brain Res 632:325-328.

Gavalas A, Krumlauf R (2000) Retinoid signalling and hindbrain patterning. Curr Opin Genet Dev 10:380-386.

Ge WP, Yang XJ, Zhang Z, Wang HK, Shen W, Deng QD, Duan S (2006)
Long-term potentiation of neuron-glia synapses mediated by $\mathrm{Ca}^{2+}$ permeable AMPA receptors. Science 312:1533-1537.

Hell JW, Westenbroek RE, Warner C, Ahlijanian MK, Prystay W, Gilbert MM, Snutch TP, Catterall WA (1993) Identification and differential subcellular localization of the neuronal class C and class D L-type calcium channel alpha 1 subunits. J Cell Biol 123:949-962.

Hockerman GH, Dilmac N, Scheuer T, Catterall WA (2000) Molecular determinants of diltiazem block in domains IIIS6 and IVS6 of L-type $\mathrm{Ca}^{2+}$ channels. Mol Pharmacol 58:1264-1270.

Jakawich SK, Nasser HB, Strong MJ, McCartney AJ, Perez AS, Rakesh N, Carruthers CJ, Sutton MA (2010) Local presynaptic activity gates homeostatic changes in presynaptic function driven by dendritic BDNF synthesis. Neuron 68:1143-1158.

Ju W, Morishita W, Tsui J, Gaietta G, Deerinck TJ, Adams SR, Garner CC, Tsien RY, Ellisman MH, Malenka RC (2004) Activity-dependent regulation of dendritic synthesis and trafficking of AMPA receptors. Nat Neurosci 7:244-253.

Lin SC, Bergles DE (2004) Synaptic signaling between neurons and glia. Glia 47:290-298.

Lindskog M, Li L, Groth RD, Poburko D, Thiagarajan TC, Han X, Tsien RW (2010) Postsynaptic GluAl enables acute retrograde enhancement of presynaptic function to coordinate adaptation to synaptic inactivity. Proc Natl Acad Sci U S A 107:21806-21811.

Maghsoodi B, Poon MM, Nam CI, Aoto J, Ting P, Chen L (2008) Retinoic acid regulates RARa-mediated control of translation in dendritic RNA granules during homeostatic synaptic plasticity. Proc Natl Acad Sci U S A 105:16015-16020.

Nam CI, Chen L (2005) Postsynaptic assembly induced by neurexinneuroligin interaction and neurotransmitter. Proc Natl Acad Sci U S A 102:6137-6142.

Niederreither K, Fraulob V, Garnier JM, Chambon P, Dollé P (2002) Differential expression of retinoic acid-synthesizing (RALDH) enzymes during fetal development and organ differentiation in the mouse. Mech Dev 110:165-171.

Pang ZP, Xu W, Cao P, Südhof TC (2010) Calmodulin suppresses synaptotagmin-2 transcription in cortical neurons. J Biol Chem 285:33930-33939.

Poon MM, Chen L (2008) Retinoic acid-gated sequence-specific translational control by RARalpha. Proc Natl Acad Sci U S A 105:20303-20308.

Russo JE, Hauguitz D, Hilton J (1988) Inhibition of mouse cytosolic aldehyde dehydrogenase by 4 -(diethylamino)benzaldehyde. Biochem Pharmacol 37:1639-1642.

Schiller J, Schiller Y, Clapham DE (1998) NMDA receptors amplify calcium influx into dendritic spines during associative pre- and postsynaptic activation. Nat Neurosci 1:114-118.

Schoups AA, Elliott RC, Friedman WJ, Black IB (1995) NGF and BDNF are differentially modulated by visual experience in the developing geniculocortical pathway. Brain Res Dev Brain Res 86:326-334.

Smith D, Wagner E, Koul O, McCaffery P, Dräger UC (2001) Retinoic acid synthesis for the developing telencephalon. Cereb Cortex 11:894-905.

Soden ME, Chen L (2010) Fragile X protein FMRP is required for homeostatic plasticity and regulation of synaptic strength by retinoic acid. J Neurosci 30:16910-16921.

Sutton MA, Ito HT, Cressy P, Kempf C, Woo JC, Schuman EM (2006) Miniature neurotransmission stabilizes synaptic function via tonic suppression of local dendritic protein synthesis. Cell 125:785-799.

Thiagarajan TC, Lindskog M, Tsien RW (2005) Adaptation to synaptic inactivity in hippocampal neurons. Neuron 47:725-737.

Turrigiano GG, Nelson SB (2004) Homeostatic plasticity in the developing nervous system. Nat Rev Neurosci 5:97-107.

Turrigiano GG, Leslie KR, Desai NS, Rutherford LC, Nelson SB (1998) Activity-dependent scaling of quantal amplitude in neocortical neurons. Nature 391:892-896.

Wagner E, Luo T, Dräger UC (2002) Retinoic acid synthesis in the postnatal mouse brain marks distinct developmental stages and functional systems. Cereb Cortex 12:1244-1253.

Wang C, Kane MA, Napoli JL (2011) Multiple retinol and retinal dehydrogenases catalyze all-trans-retinoic acid biosynthesis in astrocytes. J Biol Chem 286:6542-6553.

Werner EA, Deluca HF (2002) Retinoic acid is detected at relatively high levels in the CNS of adult rats. Am J Physiol Endocrinol Metab 282:E672-E678.

Wierenga CJ, Ibata K, Turrigiano GG (2005) Postsynaptic expression of homeostatic plasticity at neocortical synapses. J Neurosci 25:2895-2905. 